\title{
Review of meta-analyses evaluating surrogate endpoints for overall survival in oncology
}

This article was published in the following Dove Press journal:

OncoTargets and Therapy

19 October 2012

Number of times this article has been viewed

\section{Beth Sherrill' \\ James A Kaye ${ }^{2}$ \\ Rickard Sandin ${ }^{3}$ \\ Joseph C Cappelleri ${ }^{4}$ \\ Connie Chen ${ }^{5}$}

'RTI Health Solutions, Biometrics, Research Triangle Park, NC, USA; ${ }^{2}$ RTI Health Solutions, Epidemiology, Research Triangle Park, NC, USA; ${ }^{3}$ Pfizer, Global Outcomes Research Sollentuna, Sweden; ${ }^{4}$ Pfizer,

Biostatistics, Groton, CT, USA; ${ }^{5}$ Pfizer, Global Outcomes Research New York, NY, USA
Correspondence: Beth Sherrill

3040 Cornwallis Road, PO Box 12194 ,

Research Triangle Park, NC,

27709-2194, USA

$\mathrm{Tel}+|91954| 1233$

Email bsherrill@rti.org

\begin{abstract}
Overall survival (OS) is the gold standard in measuring the treatment effect of new drug therapies for cancer. However, practical factors may preclude the collection of unconfounded OS data, and surrogate endpoints are often used instead. Meta-analyses have been widely used for the validation of surrogate endpoints, specifically in oncology. This research reviewed published meta-analyses on the types of surrogate measures used in oncology studies and examined the extent of correlation between surrogate endpoints and OS for different cancer types. A search was conducted in October 2010 to compile available published evidence in the English language for the validation of disease progression-related endpoints as surrogates of OS, based on meta-analyses. We summarize published meta-analyses that quantified the correlation between progression-based endpoints and OS for multiple advanced solid-tumor types. We also discuss issues that affect the interpretation of these findings. Progression-free survival is the most commonly used surrogate measure in studies of advanced solid tumors, and correlation with OS is reported for a limited number of cancer types. Given the increased use of crossover in trials and the availability of second-/third-line treatment options available to patients after progression, it will become increasingly more difficult to establish correlation between effects on progression-free survival and OS in additional tumor types.
\end{abstract}

Keywords: progression endpoints, correlation, cancer

\section{Introduction}

Rapid changes in our understanding of cancer biology and genetics, accompanied by the advent of newer targeted agents, are affecting every level of drug development, including molecule screening, development planning, study designs, regulatory decision making, and reimbursement choices. Although overall survival (OS) remains the gold standard for assessing patient benefit from new drug therapies for cancer, practical factors may preclude the collection of unconfounded OS data. Showing a survival advantage of one treatment over another in cancer clinical trials can take years, and if patients take other treatments that improve survival after disease progression, attributing benefits confidently to a single agent or designing a feasible trial protocol with enough patients and duration of follow-up may not be possible. In addition, the length of survival post-progression may make it difficult to detect a survival advantage, even if one exists, due to the random variation associated with patient heterogeneity and the influences of subsequent therapy. ${ }^{1}$ An obvious need exists for well-defined and valid measures of benefit from anticancer treatment that can be assessed earlier in the course of the disease than patient death. Since approval and access to a new product hinges on successful Phase 3 clinical trial results, surrogate endpoints that 
could support earlier decision making would provide patients with new treatments sooner and reduce the costs of drug development, as has been seen in many other therapy areas (eg, HIV/AIDS and cardiovascular disease).

A surrogate endpoint in a clinical trial is "a substitute for a clinically meaningful endpoint that measures directly how a patient feels, functions or survives."'2 A surrogate endpoint must be clinically relevant, sensitive to treatment, and measurable. ${ }^{3}$ Surrogates are particularly valuable for drug development in diseases where increased patient survival is the goal of treatment, but a long time is required to observe this endpoint directly. For example, in studies of antihypertensives, blood pressure reduction is generally accepted as a surrogate endpoint for the reduction of longer-term and more severe cardiovascular endpoints. In general, justification for the use of a surrogate depends on multiple considerations that vary depending on the disease or specific cancer, drug mechanism of action, phase of development, patient subgroup, and availability of alternate treatments. For example, response rate has a role in evaluating the antitumor activity of new drugs in Phase 1 and 2 studies, but it is not recognized as an endpoint showing patient benefit in all tumors. This distinction is partly based on the fact that the benefit of a partial tumor response is not necessarily outweighed by the toxicity associated with treatment; also, the proportion of patients responding is not always a valid predictor of survival or other clinical benefits. ${ }^{4}$ Time to progression (TTP), an endpoint that evaluates disease progression but censors deaths rather than counting them as events, has fallen out of favor in contemporary Phase 3 trials. Progression-free survival (PFS) is considered a more realistic assessment of treatment efficacy, since it counts both progression and deaths as part of the endpoint. ${ }^{5}$

For any stage in the drug development process, use of a surrogate endpoint rather than the target endpoint may shorten clinical trials but increase the chance of false positive results. ${ }^{6}$ Validation of surrogate endpoints is typically based on the Prentice criterion, ${ }^{7}$ a set of conditions that specify the relationship between the treatment and endpoints under consideration. Changes in a surrogate endpoint that are induced by a therapy are expected to reflect changes in a clinically meaningful target endpoint.

During the past decade, a body of work has developed that uses meta-analytic techniques to investigate progression-related endpoints as possible surrogates for overall survival in patients with solid tumors. ${ }^{8-12}$ The meta-analyses conducted for surrogate endpoint validation in oncology are somewhat atypical in that the objective is to establish the relationships between endpoints, rather than summarizing treatment effects on a single endpoint. To accomplish this goal, investigators use a technique called meta-regression to model a treatment effect for survival against a treatment effect for the potential surrogate endpoint. For example, based on individual-patient data or summary data from multiple clinical trials, the hazard ratio (HR) for comparing two treatments on overall survival (HRos) can be regressed on the hazard ratio for PFS (HRpfs), resulting in an equation such as the following:

$$
\operatorname{HRos}_{\mathrm{i}}=\mu+\left(ß \times \operatorname{HRpfs}_{\mathrm{i}}\right)+\dot{\varepsilon}
$$

where $\mu$ represents an intercept, $\beta$ is the slope of the line showing the linear relationship of the hazard ratios, and $\varepsilon$ is the unexplained variance. In Equation (1), each study contributes one observation, typically weighted by the variance of the study-specific HR. Such an analysis expresses the relationship between differences in effect sizes for progression and survival across multiple trials and gives an idea of how strongly the endpoints are linked mathematically, assuming a linear relationship.

In other words, the meta-regression equation shows the predicted relationship between the hazard ratios for progression-free survival and overall survival, based on the studies included. If the slope $(ß)$ of this equation equals 1 , assuming a negligible intercept, the treatment effects on survival are expected to be of similar magnitude to effects on PFS. Models may address covariates or factors that can influence the endpoint relationship, and sometimes the metaanalysis is repeated on different patient subgroups or subsets of studies. Meta-regression equations take many different forms in the published literature, depending on factors such as which endpoint was evaluated, whether a transformation (logarithm) was used, what statistical model was implemented, and how study weights were derived. Some authors model the difference between treatments in median months to the event as the treatment effect, or analyze data from the study arms separately.

Typically, authors present the simple correlation $r$ between the treatment effect measures across trials. Correlation values are close to one if the treatment effects tend to go in the same direction. In other words, correlation is high if the hazard ratios for PFS and OS are similar across trials; correlation is low if the hazard ratios are unrelated or in opposite directions. A related measure ( $\mathrm{R}^{2}$ or $\mathrm{R}$-squared) is derived from the meta-regression equation to indicate how much variance 
in OS is explained by the potential surrogate PFS. In the very simplest case, $\mathrm{R}^{2}$ is equal to a squared correlation estimate (ie, $\mathrm{R}^{2}=\mathrm{r} \times \mathrm{r}$ ). Some authors denote whether $\mathrm{R}^{2}$ is based on (a) models of median OS and median PFS from individual study arms $\left(\mathrm{R}^{2}{ }_{\text {ind }}\right)$ or (b) models of hazard ratio for OS and hazard ratio for PFS $\left(\mathrm{R}_{\text {trial }}^{2}\right)$ (Figure 1). Otherwise, the publication may simply show $\mathrm{r}$ or $\mathrm{R}^{2}$ and leave it to the reader to distinguish which one is being used. Because $\mathrm{R}_{\text {trial }}^{2}$ addresses whether treatment effects on the surrogate endpoint are associated with treatment effects on survival, it is particularly important for drug development.

While many authors have presented meta-analyses of the relationship between treatment effects on PFS and OS in specific cancers, we have not seen a compilation of this evidence across tumor types. In October 2010, we reviewed published meta-analyses used for the validation of progression-related surrogate endpoints in multiple tumor types with the goal of summarizing the existing evidence, assessing differences and similarities across tumor types, and elucidating the assorted challenges associated with this topic. We conducted a comprehensive and iterative review of the literature, identifying more than 1000 titles and scanning more than 100 full-text articles for relevance, including review articles, methods papers, and commentary from clinicians, statisticians, regulators, and payers. We focused on evidence on disease-progression endpoints that was likely to be used in Phase 3 studies conducted for regulatory approval of new treatments for patients with solid tumors, especially in the advanced/metastatic setting. Relevant papers that were published while this manuscript was in preparation were also incorporated.

Multiple solid tumors were represented in publications (Table 1) that were identified as using meta-analyses to evaluate the following progression-related endpoints as surrogates for $\mathrm{OS}$ :

- Disease-free survival - adjuvant setting only - or eventfree survival

- Response rate or objective response rate
- TTP

- PFS

Many articles presented a meta-regression showing the relationship between treatment effects on these endpoints and treatment effects on OS, but we found little consistency in the model form or presentation of predicted values.

\section{Summary of available evidence by cancer type Colorectal cancer}

Colorectal cancer is currently the only metastatic tumor type for which consistently strong validation evidence is available on progression-free survival as a surrogate for overall survival. A number of published papers that examined progression-related endpoints as potential surrogates for survival in colorectal cancer presented graphs with regression equations for the relationship between treatment effects on the surrogate endpoint and on survival. Studies with results falling almost perfectly on a diagonal line to depict the relationship between hazard ratios for disease-free survival and overall survival contributed to the acceptance of this endpoint as a valid surrogate endpoint in the adjuvant treatment setting for colorectal cancer. ${ }^{13,14}$

The published meta- analyses provide strong evidence for the correspondence between progression-free survival and overall survival in the metastatic disease setting, although the equations predict that hazard ratios for overall survival will be somewhat closer to the null than hazard ratios for progression-free survival. Tang and colleagues ${ }^{15}$ concluded that a novel therapy producing a $10 \%$ risk reduction for progression-free survival would yield an estimated 5.4\% improvement in overall survival. Buyse and colleagues ${ }^{16}$ predicted that risk reductions in colorectal cancer would be lower on overall survival than on progression-free survival, and they suggested a threshold effect that a new treatment would have to show for the hazard ratio for

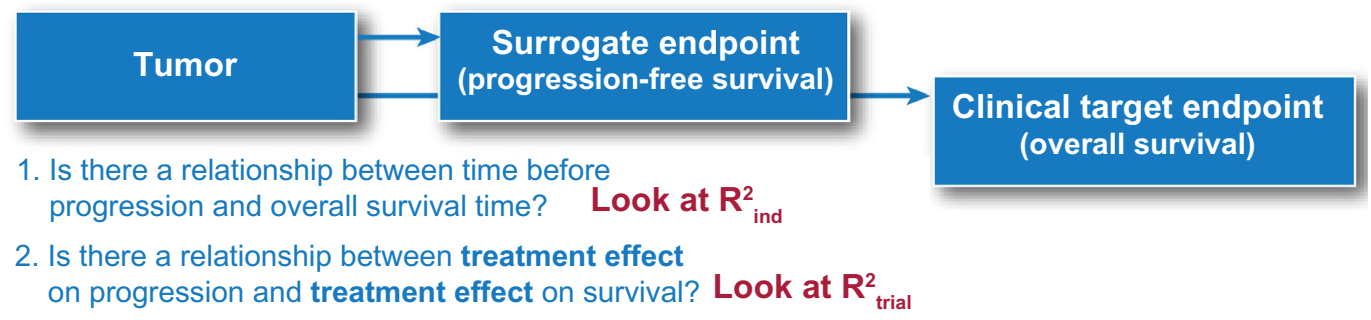

Figure I Diagram of the relationship between treatment and PFS as potential surrogate endpoint for OS.

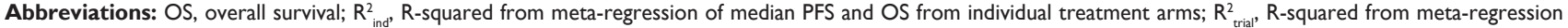
of hazard ratios from each trial. 
Table I Meta-analytic evidence for correlation between progression endpoints and OS in multiple solid tumors as reported in publications

\begin{tabular}{llll}
\hline Cancer setting & Surrogate & $\begin{array}{l}\text { Correlation between } \\
\text { surrogate and OS endpoints }\end{array}$ & $\begin{array}{l}\text { Correlation between treatment } \\
\text { effects on surrogate and on OS }\end{array}$
\end{tabular}

\section{A}

Colon, adjuvant

Colorectal, adjuvant

Colorectal, advanced

Colorectal, metastatic

Colorectal, metastatic

Colorectal, metastatic

Colorectal, metastatic

Colorectal, metastatic

Colorectal, advanced

Colorectal, metastatic

Colorectal, metastatic

Colon or colorectal,

metastatic

B

Ovarian, advanced

Ovarian, metastatic

Ovarian: platinum-resistant

Ovarian: advanced

C

Breast, adjuvant

Breast, metastatic

Breast, metastatic

Breast, metastatic

Breast, advanced

Breast, advanced

Breast, metastatic

Breast, metastatic

Breast, metastatic

PFS

\section{D}

NSCLC

NSCLC, advanced

NSCLC

SCLC

E

Brain (glioblastoma multiforme)

Head and neck, locally advanced

(radiotherapy trials)

Prostate, castrate-resistant PFS

Prostate, advanced PFS

Renal cell carcinoma PFS

Multiple metastatic solid tumors: breast, PFS

pancreatic, colon or colorectal, ovarian,

DFS

TTP

PFS

PFS

$\mathrm{PFS}^{\mathrm{b}}$

EFS

\begin{tabular}{|c|c|c|}
\hline DFS & $R: 0.88 ; R^{2}: 0.85$ & $R: 0.94 ; R^{2}: 0.90$ \\
\hline DFS & & $R: 0.95$ \\
\hline Response rate & & $R^{2}: 0.38$ \\
\hline Response rate & R: 0.408 & \\
\hline Response rate & R: 0.59 & \\
\hline Response rate & & $R^{2}: 0.10$ \\
\hline TTP & & $R^{2}: 0.33$ \\
\hline TTP & $R: 0.24$ & \\
\hline PFS & $R: 0.82$ & R: $0.99 ; R: 0.74$ (sensitivity analysis) \\
\hline PFS & R: 0.79 & Difference in PFS $R^{2}: 0.65$ \\
\hline PFS & $R: 0.48 \mathrm{I}$ & \\
\hline PFS & & $\begin{array}{l}\text { Difference in PFS R2: } 0.64 \text {; } \\
\text { HR PFS } R^{2}: 0.52\end{array}$ \\
\hline
\end{tabular}

TTP $\quad \mathrm{R}_{\text {ind }}^{2}: 0.88$

PFS At 6 months, R: 0.66

PFS $\quad R^{2}: 0.70$

ORR

Response rate

TTP R: 0.682

PFS R: 0.688

TTP, PFS TTP and PFS: R: 0.38

Response rate

Response rate a

TTP

Kappa statistics:

0.48 to 0.52

R: 0.86

Association 0.30
$\mathrm{R}^{2}$ trial: 0.94

Difference in PFS R $R^{2}: 0.60$;

HR PFS R²: 0.73

$R^{2}: 0.95$

$R^{2}: 0.38$ (only DFS), 0.39 (node \pm ),

0.37 (hormone trials), 0.43

(chemotherapy trials)

Difference in ORR $R^{2}: 0.20$;

HR ORR R2: 0.10

R: 0.57

R: 0.49

$R^{2}: 0.67$ for trials before 1990

$R^{2}$ : 0.4 I for trials after 1990

Anthracyclines, $R^{2}: 0.49$

Taxanes, $R^{2}: 0.35$

R: 0.48

Overall R²: 0.30; HR PFS R²: 0.52;

Anthracyclines $R^{2}$ : 0.43; hormonal

$R^{2}: 0.24$; HER2+ $R^{2}: 0.93$

Difference in PFS R': 0.30;

HR PFS R $2: 0.78$

$\begin{array}{ll}R^{2}: 0.16 & 29 \\ & 63 \\ R^{2}: 0.19 & 29 \\ R^{2}: 0.79 & 28\end{array}$

$\begin{array}{ll}\mathrm{R}: & 0.53 \quad 39\end{array}$

$\begin{array}{ll}\mathrm{R}: 0.98 & 38\end{array}$

$R^{2}: 0.22$

Differences in progression

R: $0.69 ; R^{2}: 0.46$

Overall difference in PFS

$R^{2}$ : 0.49; HR PFS R²: 0.62
9,13

4

76

5

29

29

16

5

17

57

58

57

59

60

22

61

62

62

25

24

62

23

57

29

63

29

37

8

34

57

renal cell carcinoma, esophago-gastric

Notes: ${ }^{2}$ Author noted significant relationship $(P<0.05)$ but did not provide $R$ or $\mathrm{R}^{2}$; blooked at proportion with progression at 6 months associated with overall survival at 12 months.

Abbreviations: OS, overall survival; DFS ${ }^{\circledR}$, disease-free survival; EFS, event-free survival; NSCLC, non-small cell lung cancer; ORR, objective response rate; PFS, progressionfree survival; SCLC, small cell lung cancer; TTP, time to progression; $R^{2}$, R-squared. 
progression-free survival $\left(\mathrm{HR}_{\mathrm{PFS}}\right)$ to have a beneficial impact on overall survival.

The evidence led to a consensus among researchers that these endpoints were valid surrogates for survival in studies of colorectal cancer. ${ }^{15-17}$ On the other hand, analyses referenced in Table 1A also helped to establish that response rate and time to progression were insufficient as surrogate endpoints for survival in colorectal cancer studies.

\section{Ovarian cancer}

As long ago as 1992, Torri et a ${ }^{18}$ presented a correlation of endpoints in advanced ovarian cancer by separate treatment arms. The evidence we compiled recommends progressionfree survival or even the time to progression as a surrogate (Table 1B). However, researchers have found the data supporting the validity of progression-free survival as a surrogate for overall survival in second- and third-line therapy to be less clear than those for first-line therapy. ${ }^{19,20}$

Molenberghs and colleagues ${ }^{21}$ compared various surrogacy criteria and concluded that progression-free survival was not a useful endpoint in ovarian cancer because it could take a long time for ovarian cancer to cause symptoms or to be detected by physical examination or imaging studies, and that progression was typically followed by death within a few months. Bast and colleagues ${ }^{19}$ summarized expert commentary from a Food and Drug Administration (FDA) workshop on evaluating potential endpoints in ovarian cancer drug research:

PFS seems to correlate with OS, especially when a large effect on PFS is seen. A small increase in PFS may not correlate with OS, however, and crossover confounds the measurement of OS.

However, PFS has gained wide acceptance as a suitable surrogate endpoint in Phase 3 studies for the first-line treatment of metastatic ovarian cancer. Further evidence is needed to support its use in resistant or refractory disease.

\section{Breast cancer}

Findings from meta-regressions of breast cancer studies are less convincing than those of other tumors; two authors provided predictions indicating that the treatment effect on overall survival was expected to be much smaller than on the progression endpoint in trials of breast cancer, whether in the adjuvant ${ }^{22}$ or advanced setting. ${ }^{23}$ Correlations $\left(\mathrm{R}_{\text {ind }}{ }^{2}\right)$ between progression-free survival or time-to-progression endpoints and survival ranged from 0.38 to 0.68 when individual treatment arms were analyzed (Table 1C). The association of treatment effects $\left(\mathrm{R}_{\text {treat }}^{2}\right)$ on these endpoints varies widely, ranging from 0.24 to 0.78 , and it is not clear whether the variability is due to different treatment types, line of therapy, frequency of crossover or use of other therapies after progression, data quality, analytical approach, or other factors.

Several of the published meta-analyses of surrogacy in breast cancer present analyses of subgroups, in which authors investigated whether a stronger relationship between endpoints may be apparent in certain trial settings. Results seem to vary by which drug class was studied and whether individual-patient data are available for analysis; in other words, the subgroup analyses have generally uncovered mixed information, making it difficult to draw definite conclusions. Miksad and colleagues ${ }^{24}$ reported regression equations that implied a stronger relationship between endpoints for studies using taxane-based rather than anthracycline-based chemotherapies, so in studies of breast cancer, the specific treatment evaluated may affect the correlation between a surrogate endpoint and overall survival. Both Miksad and colleagues ${ }^{24}$ and Hackshaw and colleagues ${ }^{25}$ showed higher $\mathrm{R}^{2}$ values between the hazard ratios for progression-free survival and overall survival for trials conducted prior to 1990. A possible explanation for this finding is that increased availability of second-line therapies for patients in more recent trials obscures the relationships between survival and the surrogate endpoints.

Authors of these meta-analyses and others who have commented on these results have generally considered the evidence inadequate to fully support the use of progression endpoints as surrogates for survival in breast cancer studies; the primary concerns are that the correlations are too weak and the predictions are too uncertain..$^{22-24,26,27}$

\section{Lung cancer}

Correlations between response rate or TTP and the survival outcome in non-small cell lung cancer (NSCLC) were low, but evidence supports PFS as a surrogate measure in extensive-stage small cell lung cancer (SCLC). ${ }^{28}$ Several published meta-analyses in NSCLC provide predictions for OS treatment effects based on the effects of treatment on the response rate or time to progression, but the correlation values for these analyses are extremely low (Table 1D). Johnson and colleagues ${ }^{29}$ also examined factors that might help explain the relationship between effects on the surrogate endpoint 
and survival, such as patient age, performance status, stage of disease, year of trial, and use of rescue (or salvage) treatment, but did not identify any other factor that predicted differences in survival.

A meta-analysis of six single-arm and three randomized trials in patients with extensive-stage SCLC showed correlation between PFS and OS as strong as that seen in colorectal studies. ${ }^{28}$ However, the authors of this study cautioned that further validation, using data from a larger number of randomized Phase 3 trials, was needed. The apparently better performance of PFS as a surrogate for OS in SCLC than in NSCLC may relate to the more aggressive untreated clinical course of SCLC compared with NSCLC and the higher responsiveness and greater proportional survival benefit with systemic treatment (at least 4 - to 5-fold) ${ }^{30}$ for patients with SCLC, compared to those with NSCLC (approximately 33\% improvement in median survival, from 4.5 to 6 months). ${ }^{31}$

\section{Renal cell carcinoma}

Several approaches have been used to assess the relationship between progression and survival in renal cell carcinoma. Two groups of investigators reviewed results from clinical trials and reported an association between progression-free survival and overall survival, ${ }^{32,33}$ without presenting any analyses to validate progression-free survival as a surrogate endpoint. Kane and colleagues ${ }^{33}$ presented their conclusion as support for the approval of sorafenib for the treatment of renal cell carcinoma largely due to the persuasive magnitude of the improvement in progression-free survival.

At the 2009 annual meeting of the American Society of Clinical Oncology, Delea and colleagues ${ }^{34}$ presented a metaanalysis of 21 trials of treatments for renal cell carcinoma; they reported strong correlation $(r=0.69)$ between group differences in median time to progression-free survival and overall survival. The results suggested that a 1-month difference in disease progression was associated with a 1.4-month difference in overall survival. This work was cited in other publications and by the Australian Pharmaceutical Benefits Advisory Committee (PBAC) ${ }^{35}$ in its decision regarding everolimus. PBAC stated that Delea's meta-analysis might not be generalizable to the specific context where crossover to the new treatment was prevalent and rapid, and also noted the lack of information in the abstract regarding a surrogate threshold-effect size.

Rather than using meta-analysis, Heng et $\mathrm{al}^{36}$ used landmark analysis to suggest that PFS at 3 and 6 months can predict survival. However, the effect of early progressors was not factored into these results, since the landmark analysis excluded patients who progressed or died before the landmark timepoint.

\section{Other cancers}

In prostate cancer studies, low observed correlations between PFS and OS suggest that PFS is not a useful surrogate for OS in this disease (Table 1E). Progression-free survival has been evaluated as a surrogate for survival in studies of prostate cancer, but the low correlations do not recommend its use for this purpose. ${ }^{8,37}$

One publication reported strong correlation between event-free survival and OS in head and neck cancer. ${ }^{38}$ This meta-analysis provided evidence that event-free survival, defined as time from randomization to locoregional, distant recurrence, or death from any cause, could be used as a surrogate for overall survival in patients with locally advanced head and neck cancer. The analysis was based on a heterogeneous group of clinical trials in which patients were treated with radiotherapy and/or with concomitant, induction, or adjuvant chemotherapy.

One publication reported moderate correlation between 6-month PFS and 1-year OS in patients with glioblastoma multiforme. ${ }^{39}$ Recent publications include meta-analyses evaluating PFS as a surrogate for OS in advanced gastric cancer $^{40}$ and non-Hodgkin's lymphoma; ${ }^{41}$ a meta-analysis of surrogates in metastatic melanoma came to our attention after the initial literature search. ${ }^{42}$

\section{Discussion}

PFS is the most commonly used surrogate measure in studies of advanced solid tumors, but is not universally accepted, and evidence for its validity varies by tumor type. Correlation with OS is reported for a limited number of cancer types, and validation findings vary by the specific cancer indication, patient subgroups, analytical approaches, and the effectiveness of treatments being studied. In summary, published meta-analyses provide good evidence for the use of PFS as a surrogate for overall survival in advanced colorectal or ovarian cancer. Evidence in other cancers is limited. In breast cancer, for example, a number of papers have examined the topic of surrogate endpoints using meta-analyses, but the variation in findings is not well understood.

A real treatment effect on a progression endpoint may not predict an effect on overall survival for a number of reasons. It remains possible that progression is not a viable surrogate in some cancers, such as prostate cancer. Even when it is suitable as a surrogate, the relationship between treatment 
effects on progression and survival are expected to change in newer trials as more-effective treatments emerge or as new mechanisms of action are explored. The issues discussed here contribute to the difficulty of validating progression-related endpoints as surrogates for OS in oncology studies, and partly explain the lack of evidence in some tumor types.

\section{Study design issues}

Determination of progression endpoints depends on the definition applied, the frequency and methods of monitoring, evaluator objectivity, and the number and location of lesions that are evaluated. For example, if scans to assess progression in a particular trial are performed every 6 months, then the earliest that progression can be documented is at 6 months; whereas if scans are done every 2 months, then progression can be seen earlier. ${ }^{43}$ Thus, studies that have different assessment schedules may show very different relationships between progression timing and overall survival, even if the treatment effects on both endpoints were identical.

Several years and large sample sizes are usually required to collect survival data. Patients often take additional anticancer treatments after disease progression; if effective, these subsequent treatments extend survival. Use of subsequent treatments confounds the effects of the initial treatment of interest and introduces variability, making it harder to pick up a survival signal. When large advantages are identified for a new treatment, ethical considerations prompt offering it to all patients, even those in the control arm. Perversely, this means that we may not be able to observe unconfounded overall survival data for treatments that show significant effects on progression. If treatment administered after progression confounds the association, or if survival after progression is relatively long compared with time to progression, then postprogression factors (eg, noncancer deaths, declining sample size, variability in supportive care) may outweigh treatment differences in time to progression. In other cases, the magnitude of improvement in progression-free survival may be insufficient to translate into a survival benefit.

\section{Clinical and biological issues}

Even when no further anticancer treatment is given after progression, individual patient variation in tumor growth may obscure the relation of tumor response to survival duration. Many traditional cytotoxic chemotherapy agents are mutagenic, and clonal evolution in cancers is a well-documented phenomenon, ${ }^{44,45}$ so it is theoretically possible that clonal evolution could be accelerated by exposure to cytotoxic drugs, which might affect the relation between PFS and survival.
Genetic variants in patients and/or tumors can influence treatment response, risk of progression, or survival. ${ }^{46,47}$

The relationship between progression and overall survival might be more apparent when we are able to select patients who are the most likely to benefit from a particular treatment, such as when a targeted therapy is directed against a mutated protein that occurs in the tumor cells in only a proportion of patients. Until reliable predictive markers and effective targeted therapies are available for most patients with a given type of cancer, we will need to repeatedly reassess the existing evidence in light of new discoveries. Additionally, some targeted therapies, such as those directed at angiogenesis, may induce disease stabilization rather than tumor regression; therefore, tumors may not exhibit the same pattern of disease progression as that seen under treatment with older regimens. It remains to be seen whether endpoints such as progression-free survival can adequately capture the benefits of these drugs for patients.

\section{Statistical and validation issues}

Even if a treatment extends survival by the same amount as it extends time to progression, the relationship between endpoints could be difficult to portray statistically. For the purposes of evaluating the validity of a surrogate endpoint using meta-analysis, evidence must be available from similar trials that measured survival without confounding; for a new drug or a new indication, there may not be enough previously conducted trials to conduct such an analysis. Meta-analysis results are affected by which studies are included, what endpoints are evaluated, and what analytical methods are used to model the treatment effects. There is no consensus on what level of correlation between hazard ratios is required to consider PFS a useful surrogate for OS. Furthermore, even if the PFS hazard ratio could be used to predict the OS hazard ratio, it would not convey information on how long patients are expected to live. The hazard ratio depicts differences in the probability of the event over time between two groups, but the magnitude of difference in time to event between treatment groups must also be considered when assessing benefit.

Although meta-analysis has been used extensively for showing the relationship between oncology endpoints, a conceptual difficulty arises from the fact that PFS and OS definitions overlap (ie, preprogression time is a subset of OS). This inherent dependency between PFS and OS has typically not been addressed in meta-regressions, but recent methodological developments account for the dependency structure between OS and PFS (eg, by relating 
PFS to post-progression survival). Some of these promising approaches include specialized parametric models, ${ }^{48,49}$ simulations, ${ }^{1,50}$ and sophisticated multistate models of joint distribution of endpoints that incorporate the time between progression and survival when making predictions. ${ }^{51,52}$

\section{Regulatory issues}

In general, justification for the use of a surrogate endpoint for drug approval depends on such considerations as tumor type, patient population, line of treatment, drug mechanism of action, development phase, and availability of alternate treatments. The European Medicines Agency has accepted PFS and disease-free survival as primary endpoints in situations where there is a "large effect on progression-free survival, a long expected survival after progression, or a clearly favorable safety profile." ${ }^{53}$ In refractory metastatic tumors or when no available alternative therapies exist, the FDA may grant approval based on the effect of treatment on a surrogate endpoint that is "reasonably likely to predict a clinical benefit." ${ }^{54}$ Draft guidance from the FDA for endpoints in NSCLC states that consideration of PFS as an endpoint for the demonstration of efficacy for drug approval will be based on the magnitude of the effect and the risk-benefit profile of the drug. "Because of the subjectivity in the measurement of PFS assessments and the fact that assessments depend on frequency, accuracy, reproducibility, and completeness, the observed magnitude of effect should be substantial and robust." ${ }_{55}$ In other words, regardless of evidence for the validity of PFS as a surrogate for survival, the magnitude of benefit continues to be a key driver for the approval of new drugs.

\section{Conclusion}

The interpretation of treatment differences, however expressed, requires some judgment, and the threshold defining a clinically important effect for different tumor types depends on many factors, such as natural history of the disease, size and duration of effect, and available alternative therapy. Given the practice of treatment crossover after disease progression, the growing availability of second- and third-line treatment options, and new drug mechanisms of action, it will become increasingly more difficult to establish the relationship between effects on PFS and OS in additional tumor types using meta-analyses based on previously conducted clinical trials. Thus, the magnitude of a treatment effect and the benefit-risk balance remain important considerations in using progression-related endpoints as surrogate endpoints for survival in oncology research. More methodological advancements that address statistical issues and related clinical interpretation are encouraged.

\section{Acknowledgments/disclosure}

This study was sponsored by Pfizer Inc. The authors wish to acknowledge Pfizer Inc, for funding the research for this manuscript. The decision to submit it was made jointly by all authors. Sherrill and Kaye are employees of RTI Health Solutions, a research organization that receives contract funding from multiple pharmaceutical companies. Chen, Capiellieri and Sandin are employees of Pfizer, Inc.

\section{References}

1. Broglio KR, Berry DA. Detecting an overall survival benefit that is derived from progression-free survival. J Natl Cancer Inst. 2009;101: 1642-1649.

2. Temple RJ. A regulatory authority's opinion about surrogate endpoints. In: Nimmo WS, Tucker GT, editors. Clinical Measurement in Drug Evaluation. New York: J Wiley; 1995. Cited in: Fleming TR, DeMets DL. Surrogate end points in clinical trials: are we being misled? Ann Intern Med. 1996;125(7):605-613.

3. Sargent D. General and statistical hierarchy of appropriate biologic endpoints. Oncology (Williston Park). 2006;20:5-9.

4. Johnson JR, Williams G, Pazdur R. End points and United States Food and Drug Administration approval of oncology drugs. J Clin Oncol. 2003;21:1404-1411.

5. Sridhara R, Johnson JR, Justice R, Keegan P, Chakravarty A, Pazdur R. Review of oncology and hematology drug product approvals at the US Food and Drug Administration between July 2005 and December 2007. J Natl Cancer Inst. 2010;102:230-243.

6. Weir CJ, Walley RJ. Statistical evaluation of biomarkers as surrogate endpoints: a literature review. Stat Med. 2006;25:183-203.

7. Prentice RL. Surrogate endpoints in clinical trials: definition and operational criteria. Stat Med. 1989;8(4):431-440. Cited in: Lassere MN. The Biomarker-Surrogacy Evaluation Schema: a review of the biomarkersurrogate literature and a proposal for a criterion-based, quantitative, multidimensional hierarchical levels of evidence schema for evaluating the status of biomarkers as surrogate endpoints. Stat Methods Med Res. 2008;17(3):303-340.

8. Buyse M. Use of meta-analysis for the validation of surrogate endpoints and biomarkers in cancer trials. Cancer J. 2009;15:421-425.

9. Buyse M. Contributions of meta-analyses based on individual patient data to therapeutic progress in colorectal cancer. Int J Clin Oncol. 2009; 14:95-101.

10. Hughes MD. Practical issues arising in an exploratory analysis evaluating progression-free survival as a surrogate endpoint for overall survival in advanced colorectal cancer. Stat Methods Med Res. 2008;17:487-495

11. Burzykowski T, Buyse M. Surrogate threshold effect: an alternative measure for meta-analytic surrogate endpoint validation. Pharm Stat. 2006;5:173-186.

12. Sargent DJ, Hayes DF. Assessing the measure of a new drug: is survival the only thing that matters? J Clin Oncol. 2008;26:1922-1923.

13. Sargent DJ, Wieand HS, Haller DG, et al. Disease-free survival versus overall survival as a primary end point for adjuvant colon cancer studies: individual patient data from 20,898 patients on 18 randomized trials. J Clin Oncol. 2005;23:8664-8670.

14. Burzykowski T, Buyse M, Yothers G, Sakamoto J, Sargent D. Exploring and validating surrogate endpoints in colorectal cancer. Lifetime Data Anal. 2008;14:54-64. 
15. Tang PA, Bentzen SM, Chen EX, Siu LL. Surrogate end points for median overall survival in metastatic colorectal cancer: literature-based analysis from 39 randomized controlled trials of first-line chemotherapy. J Clin Oncol. 2007;25:4562-4568.

16. Buyse M, Burzykowski T, Carroll K, et al. Progression-free survival is a surrogate for survival in advanced colorectal cancer. J Clin Oncol. 2007;25:5218-5224.

17. Louvet C, de Gramont A, Tournigand C, Artru P, Maindrault-Goebel F, Krulik M. Correlation between progression free survival and response rate in patients with metastatic colorectal carcinoma. Cancer. 2001;91:2033-2038.

18. Torri V, Simon R, Russek-Cohen E, Midthune D, Friedman M. Statistical model to determine the relationship of response and survival in patients with advanced ovarian cancer treated with chemotherapy. J Natl Cancer Inst. 1992;84:407-414.

19. Bast RC, Thigpen JT, Arbuck SG, et al. Clinical trial endpoints in ovarian cancer: report of an FDA/ASCO/AACR Public Workshop. Gynecol Oncol. 2007;107:173-176.

20. Vermorken JB, Parmar MK, Brady MF, et al. Clinical trials in ovarian carcinoma: study methodology. Ann Oncol. 2005;16 Suppl 8: viii20-viii29.

21. Molenberghs G, Buyse M, Geys H, Renard D, Burzykowski T, Alonso A. Statistical challenges in the evaluation of surrogate endpoints in randomized trials. Control Clin Trials. 2002;23:607-625.

22. Ng R, Pond GR, Tang PA, MacIntosh PW, Siu LL, Chen EX Correlation of changes between 2-year disease-free survival and 5-year overall survival in adjuvant breast cancer trials from 1966 to 2006. Ann Oncol. 2008;19:481-486.

23. Sherrill B, Amonkar M, Wu Y, et al. Relationship between effects on time-to-disease progression and overall survival in studies of metastatic breast cancer. Br J Cancer. 2008;99:1572-1578.

24. Miksad RA, Zietemann V, Gothe R, et al. Progression-free survival as a surrogate endpoint in advanced breast cancer. Int J Technol Assess Health Care. 2008;24:371-383.

25. Hackshaw A, Knight A, Barrett-Lee P, Leonard R. Surrogate markers and survival in women receiving first-line combination anthracycline chemotherapy for advanced breast cancer. Br J Cancer. 2005;93: 1215-1221.

26. Shi Q, Sargent DJ. Meta-analysis for the evaluation of surrogate endpoints in cancer clinical trials. Int J Clin Oncol. 2009;14:102-111.

27. Fleming TR. Objective response rate as a surrogate end point: a commentary. J Clin Oncol. 2005;23:4845-4846.

28. Foster NR, Qi Y, Shi Q, et al. Tumor response and progression-free survival as potential surrogate endpoints for overall survival in extensive stage small-cell lung cancer: findings on the basis of North Central Cancer Treatment Group trials. Cancer. 2011;117:1262-1271.

29. Johnson KR, Ringland C, Stokes BJ, et al. Response rate or time to progression as predictors of survival in trials of metastatic colorectal cancer or non-small-cell lung cancer: a meta-analysis. Lancet Oncol. 2006;7:741-746.

30. National Cancer Institute. Small cell lung cancer treatment ( $\left.P D Q{ }^{\circledR}\right) .2011$. Available from: http://www.cancer.gov/cancertopics/pdq/treatment/smallcell-lung/healthprofessional/page4. Accessed November 14, 2011.

31. NSCLC Meta-Analyses Collaborative Group. Chemotherapy in addition to supportive care improves survival in advanced non-small-cell lung cancer: a systematic review and meta-analysis of individual patient data from 16 randomized controlled trials. J Clin Oncol. 2008;26:4617-4625.

32. Knox JJ. Progression-free survival as endpoint in metastatic RCC? Lancet. 2008;372:427-429.

33. Kane RC, Farrell AT, Saber H, et al. Sorafenib for the treatment of advanced renal cell carcinoma. Clin Cancer Res. 2006;12: 7271-7278.

34. Delea TE, Khuu A, Kay A, Zheng J, Baladi JF. Association between treatment effects on disease progression (DP) endpoints and overall survival (OS) in patients with metastatic renal cell carcinoma (mRCC). J Clin Oncol. 27:15s, 2009 (suppl; abstr 5105).
35. Pharmaceutical Benefits Advisory Committee (PBAC). Public summary document for everolimus, tablets, $5 \mathrm{mg}$ and $10 \mathrm{mg}$, Afinitor ${ }^{\circledR}$. Australian Government, Department of Health and Ageing; 2010. Available from: http://www.commcarelink.health.gov.au/internet/main/publishing.nsf/ Content/pbac-psd-Everolimus-july10. Accessed January 25, 2011.

36. Heng DY, Xie W, Bjarnason GA, et al. Progression-free survival as a predictor of overall survival in metastatic renal cell carcinoma treated with contemporary targeted therapy. Cancer. 2011;117:2637-2642.

37. Halabi S, Vogelzang NJ, Ou SS, Owzar K, Archer L, Small EJ. Progression-free survival as a predictor of overall survival in men with castrate-resistant prostate cancer. J Clin Oncol. 2009;27:2766-2771.

38. Michiels S, Le Maître A, Buyse M, et al. Surrogate endpoints for overall survival in locally advanced head and neck cancer: meta-analyses of individual patient data. Lancet Oncol. 2009;10:341-350.

39. Ballman KV, Buckner JC, Brown PD, et al. The relationship between six-month progression-free survival and 12-month overall survival end points for phase II trials in patients with glioblastoma multiforme. Neuro Oncol. 2007;9:29-38.

40. Shitara K, Ikeda J, Yokota T, et al. Progression-free survival and time to progression as surrogate markers of overall survival in patients with advanced gastric cancer: analysis of 36 randomized trials. Invest New Drugs. 2011;4. Abstract.

41. Lee L, Wang L, Crump M. Identification of potential surrogate end points in randomized clinical trials of aggressive and indolent nonHodgkin's lymphoma: correlation of complete response, time-to-event and overall survival end points. Ann Oncol. 2011;22:1392-1403.

42. Korn EL, Liu PY, Lee SJ, et al. Meta-analysis of phase II cooperative group trials in metastatic stage IV melanoma to determine progressionfree and overall survival benchmarks for future phase II trials. J Clin Oncol. 2008;26:527-534.

43. Beckman M. More clinical cancer treatments judged by progressionfree rather than overall survival. J Natl Cancer Inst. 2007;99: 1068-1069.

44. van Tilborg AA, de Vries A, de Bont M, Groenfeld LE, van der Kwast TH, Zwarthoff EC. Molecular evolution of multiple recurrent cancers of the bladder. Hum Mol Genet. 2000;9:2973-2980.

45. Mora J, Cheung NK, Gerald WL. Genetic heterogeneity and clonal evolution in neuroblastoma. Br J Cancer. 2001;85:182-189.

46. Marktel S, Marin D, Foot N, et al. Chronic myeloid leukemia in chronic phase responding to imatinib: the occurrence of additional cytogenetic abnormalities predicts disease progression. Haematologica. 2003;88:260-267.

47. d'Amore F, Chan E, Iqbal J, et al. Clonal evolution in $\mathrm{t}(14 ; 18)$-positive follicular lymphoma, evidence for multiple common pathways, and frequent parallel clonal evolution. Clin Cancer Res. 2008;14: 7180-7187.

48. Fleischer F, Gaschler-Markefski B, Bluhmki E. A statistical model for the dependence between progression-free survival and overall survival. Stat Med. 2009;28:2669-2686.

49. Négrier S, Bushmakin AG, Cappelleri JC, et al. Using a Weibull parametric model for failure-time data to assess progression-free survival as a surrogate endpoint for overall survival in a trial of patients with metastatic renal cell carcinoma. Presented at the European Multidisciplinary Cancer Congress, Stockholm, Sweden, September 23-27, 2011.

50. de Gramont A, Hubbard J, Shi Q, et al. Association between disease-free survival and overall survival when survival is prolonged after recurrence in patients receiving cytotoxic adjuvant therapy for colon cancer: simulations based on the 20,800 patient ACCENT data set. J Clin Oncol. 2010;28:460-465.

51. Dejardin D, Lesaffre E, Verbeke G. Joint modeling of progressionfree survival and death in advanced cancer clinical trials. Stat Med. 2010;29:1724-1734.

52. Rondeau V, Pignon JP, Michiels S. A joint model for the dependence between clustered times to tumour progression and deaths: a metaanalysis of chemotherapy in head and neck cancer. Stat Methods Med Res. 2011 Oct 23. [Epub ahead of print]. 
53. European Medicines Agency. Committee for Medicinal Products for Human Use (CHMP). Guideline on the evaluation of anticancer medicinal products in man. 2005. Available from: http://www.ema. europa.eu/docs/en_GB/document_library/Scientific_guideline/2009/12/ WC500017748.pdf. Accessed January 13, 2011.

54. Food and Drug Administration. Guidance for industry: clinical trial endpoints for the approval of cancer drugs and biologics. Rockville, MD: US Department of Health and Human Services; 2007. Available from: http://www.fda.gov/Food/GuidanceComplianceRegulatoryInformation/ GuidanceDocuments/default.htm. Accessed October 11, 2010.

55. Food and Drug Administration. Guidance for industry [draft]: clinical trial endpoints for the approval of non-small cell lung cancer drugs and biologics. Rockville, MD: US Department of Health and Human Services; 2011. Available from: http:/www.fda.gov/Food/ GuidanceComplianceRegulatoryInformation/GuidanceDocuments/ default.htm. Accessed November 14, 2011.

56. Buyse M, Thirion P, Carlson RW, Burzykowski T, Molenberghs G, Piedbois P. Relation between tumour response to first-line chemotherapy and survival in advanced colorectal cancer: a meta-analysis. Meta-Analysis Group in Cancer. Lancet. 2000;356:373-378.

57. Wilkerson J, Fojo T. Progression-free survival is simply a measure of a drug's effect while administered and is not a surrogate for overall survival. Cancer J. 2009;15:379-385.
58. Buyse M, Molenberghs G, Burzykowski T, Renard D, Geys H. The validation of surrogate endpoints in meta-analyses of randomized experiments. Biostatistics. 2000;1:49-67.

59. Rose PG, Tian C, Bookman MA. Assessment of tumor response as a surrogate endpoint of survival in recurrent/platinum-resistant ovarian carcinoma: a Gynecologic Oncology Group study. Gynecol Oncol. 2010;117:324-329.

60. Burzykowski T, Molenberghs G, Buyse M, Geys H, Renard D. Validation of surrogate end points in multiple randomized clinical trials with failure time end points. J Roy Stat Soc C Appl Stat. 2001;50: 405-422.

61. Bruzzi P, Del Mastro L, Sormani MP, et al. Objective response to chemotherapy as a potential surrogate end point of survival in metastatic breast cancer patients. J Clin Oncol. 2005;23:5117-5125.

62. Burzykowski T, Buyse M, Piccart-Gebhart MJ, et al. Evaluation of tumor response, disease control, progression-free survival, and time to progression as potential surrogate end points in metastatic breast cancer. J Clin Oncol. 2008;26:1987-1992.

63. Tsujino K, Kawaguchi T, Kubo A, et al. Response rate is associated with prolonged survival in patients with advanced non-small cell lung cancer treated with gefitinib or erlotinib. J Thorac Oncol. 2009;4:994-1001.
OncoTargets and Therapy

\section{Publish your work in this journal}

OncoTargets and Therapy is an international, peer-reviewed, open access journal focusing on the pathological basis of all cancers, potential targets for therapy and treatment protocols employed to improve the management of cancer patients. The journal also focuses on the impact of management programs and new therapeutic agents and protocols on

\section{Dovepress}

patient perspectives such as quality of life, adherence and satisfaction The manuscript management system is completely online and includes a very quick and fair peer-review system, which is all easy to use. Visit http://www.dovepress.com/testimonials.php to read real quotes from published authors. 